\title{
Novel approach to low substrate temperature synthesis of carbon nanotubes
}

\author{
G.Y. Chen, C.H.P. POA, V. STOLOJAN AND S.R.P. SILVA \\ Nano-Electronic Centre, Advanced Technology Institute, University of Surrey, Guildford, Surrey, GU2 7XH, United Kingdom
}

\begin{abstract}
We present a novel approach, which will potentially allow for low-temperature-substrate synthesis of carbon nanotubes using direct-current plasma-enhanced chemical vapour deposition. The approach utilizes top-down plasma heating rather than conventional heating from a conventional substrate heater under the electrode. In this work, a relatively thick titanium layer is used as a thermal barrier to create a temperature gradient between the $\mathrm{Ni}$ catalyst surface and the substrate. We describe the growth properties as a function of the bias voltage and the hydrocarbon concentrations. The heating during growth is provided solely by the plasma, which is dependent only on the process conditions, which dictate the power density and the cooling of the substrate, plus now the thermal properties of the "barrier layer". This novel approach of using plasma heating and thermal barrier allows for the synthesis of carbon nanotubes at low substrate temperature conditions to be attained with suitable cooling schemes.
\end{abstract}

\section{INTRODUCTION}

Over the past few years, the growth of carbon nanotubes has been a topic of tremendous interest and activity. This is largely due to their physical characteristics such as high aspect ratios, large surface areas and ballistic electronic transport. The unique properties of nanotubes make them attractive for applications including field emission displays, interconnects for integrated circuits, high speed ballistic transistors, fuel cell electrodes and supercapacitors [[1]-[4]].

Despite the numerous techniques available for the synthesis of carbon nanotubes, only chemical vapour deposition (CVD) offers the ability to selectively deposit carbon directly onto the substrate at reduced process temperatures [[5],[6]]. The advantages of CVD make it a technique that is highly suitable for the fabrication of electronic sensors, devices, interconnects, etc [[7]-[9]]. Although the CVD technique allows lower processing temperatures, these have typically been restricted to above $500^{\circ} \mathrm{C}$. As Melechko et. al. concludes in his review on vertically aligned growth of carbon nanofibres, that although the literature indicates that the growth of carbon nanotubes and nanofibres requires a catalyst particle temperature to be high enough to provide high carbon diffusion rates, there is no such requirement for the substrate [[10]].

This suggests that, if the high temperatures required for carbon nanotube growth can be contained to the surface or near the surface region, the growth can still proceed even if the substrate is maintained at lower temperatures. One method of achieving this is to use a thermal barrier to contain the heat close to the surface, while concurrently cooling the substrate. If this approach is to be used, the heat must therefore be provided from the top (or on the surface side). At present, most research is focused on heating the substrate using a heater located below the substrate.

Recently, there have been reports of low-temperaturesynthesis of carbon nanotubes [[11]-[14]]. However, low temperature synthesis has, so far, typically resulted in amorphous or herring-bone like structures, rather than well graphitised nanotubes. These techniques however use plasma based systems which provide a situation suitable to the approach of top down heating. The combination of plasma processing and a thermal barrier would offer the possibility of low-substrate-temperature growth.

In this work, we investigate the use of a titanium layer as the thermal barrier. Titanium is selected due to its low thermal conductivity among metals, and also the restriction of using insulating materials in DC generated plasmas. The use of the titanium layer limits the transfer of heat generated by the plasma, thus maintaining a temperature difference between the surface and the substrate. The effective use of the plasma to achieve the lowest possible surface temperature for carbon nanotube growth would allow for the greater flexibility in using this approach. Besides showing that growth is possible using this novel method, the influences of the process parameters are also investigated.

\section{EXPERIMENTAL}

The PECVD system used for the growth has a parallel plate electrode configuration, driven by a direct current power source. The electrode is a 2.5 " diameter graphite plate. Using acetylene as the carbon feedstock and nitrogen/hydrogen as the carrier/dilution gases respectively, carbon nanostructures are synthesised without the application of an external heat source (other than heating from the plasma). The system base pressure was $10^{-3}$ Torr and the graphite substrate table temperature was monitored using an embedded thermocouple. The temperature reading for each run was taken as the highest temperature reached during the deposition. The concentration of hydrocarbon was simply taken as the gas flow ratio of 
acetylene to a nitrogen/hydrogen mixture. An electrode separation of $1 \mathrm{~cm}$ was used on all depositions. A $500 \mathrm{~nm}$ thick layer of titanium was first sputter-deposited onto a $\mathrm{Si}$ (100) substrate, followed by the sputter-deposition of a $30 \mathrm{~nm}-$ thick nickel layer. The sample was not pre-treated in any form before the deposition. A typical nanotube growth run would start with the backfilling of the evacuated chamber to the desired operating pressure, followed by the ignition of the plasma using the nitrogen/hydrogen mixture, and the subsequent introduction of acetylene. The sample was then cooled in an inert nitrogen ambient, before removal for subsequent characterisation. The morphology and microstructure of the nanotubes were characterised using a Hitachi S-4000 Field Emission (FE) Scanning Electron microscope (SEM) and a Philips CM200 transmission electron microscope (TEM) (200 kV accelerating voltage, $\mathrm{LaB}_{6}$ filament, line resolution of $0.14 \mathrm{~nm}$ ).

\section{RESULTS AND DISCUSSION}

The synthesis of the carbon nanotubes was carried out at 10 Torr for $45 \mathrm{~min}$, with $500 \mathrm{~V}$ of applied bias. Figure 1 shows the changes in carbon nanostructures, synthesized for hydrocarbon concentrations of 1,3 and $6 \%$.

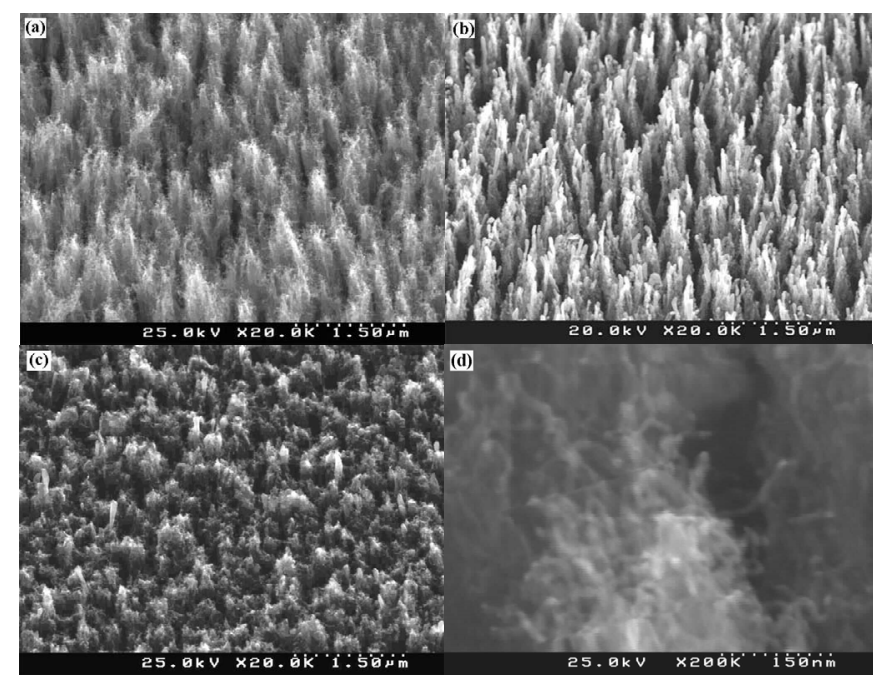

Figure 1: SEM images of the effect of different concentration of hydrocarbon concentration; (a) $1 \%$, (b) $3 \%$, (c) $6 \%$ and (d) high magnification image of fig 1a. Reducing the hydrocarbon concentration is essential for carbon nanotube at low substrate temperatures.

Although carbon nanotubes were observed for all hydrocarbon concentrations, as the hydrocarbon content is increased, the quantity of amorphous carbon increased, correspondingly. The SEM images clearly show that the control of the carbon in the system is crucial to nanotube synthesis. At the lowest hydrocarbon concentration of $1 \%$, the carbon nanotubes are formed in discrete bundles. Above 6\% hydrocarbon content, the deposit begins to resemble an amorphous carbon thin film formation [Figure 1c]. The importance of balancing the hydrocarbon concentration has always been critical, even for high-temperature growth, or with an etching gas like ammonia [[5],[16]].

Figure 2 shows the effect of changing the applied bias on the synthesis. The plasma was ignited at 10 Torr, with $3.0 \%$ hydrocarbon concentration, for $45 \mathrm{mins}$.

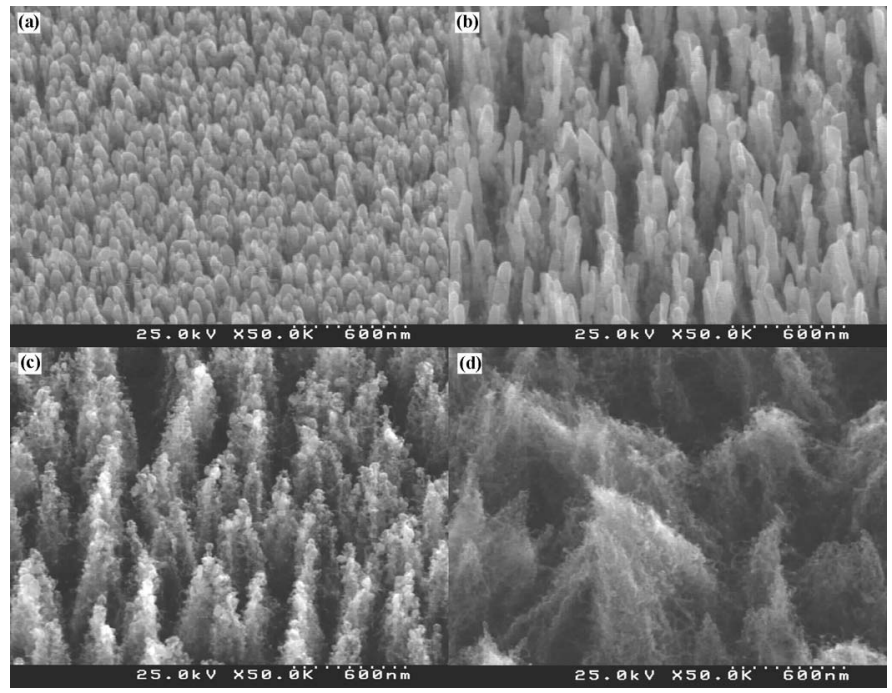

Figure 2: SEM images of the effect of different applied bias; (a) 450V, (b) $475 \mathrm{~V}$, (c) $500 \mathrm{~V}$ and (d) $550 \mathrm{~V}$.

It can be seen that, at the lowest voltage, the energy of the plasma was only sufficient in forming small carbon nanostructures. As the applied voltage increases, the structures change from pillars of sizes of around $100 \mathrm{~nm}$ to nanotube bundles. At $500 \mathrm{~V}$, nanotubes were observed, though most of the structures consisted of predominately amorphous carbon. At the highest voltage of $550 \mathrm{~V}$, the nanotubes were formed in a similar manner as in figure 1a. In all the plasma processes, the maximum recorded substrate temperature was between 350 and $417^{\circ} \mathrm{C}$. Figure 3 shows a graph of the substrate temperature monitored against the plasma bias.

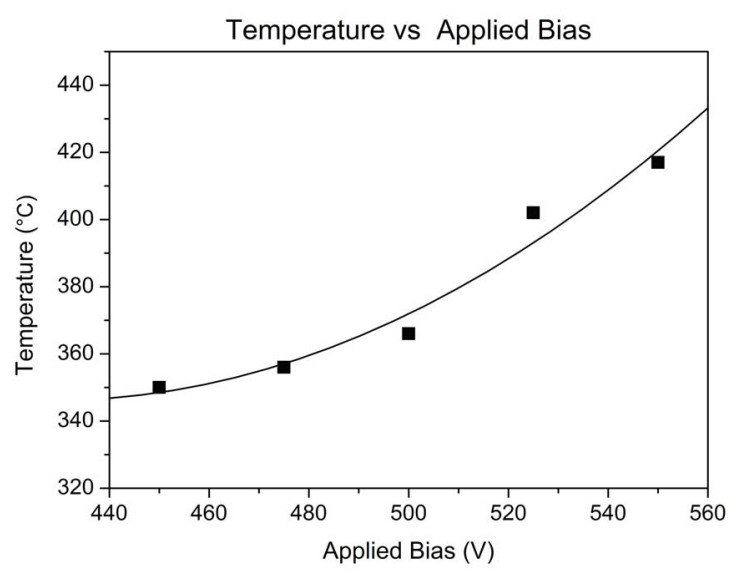

Figure 3: The relationship between the measured maximum substrate temperature and the applied plasma bias based on a 2.5 " cathode diameter. 
The substrate temperature increases with the applied plasma voltage. This is as expected, as more energy is delivered to the system. It can be noted that the substrate temperature is relatively lower than the typical synthesis temperature reported.

Nanotubes synthesised at $550 \mathrm{~V}$ were scratched off the substrate and ultrasonically dispersed in methanol. A drop of the solution was deposited on a holey carbon grid and the internal morphology of the nanotubes analysed. Figure 4 shows the TEM image of a bundle of nanotubes.

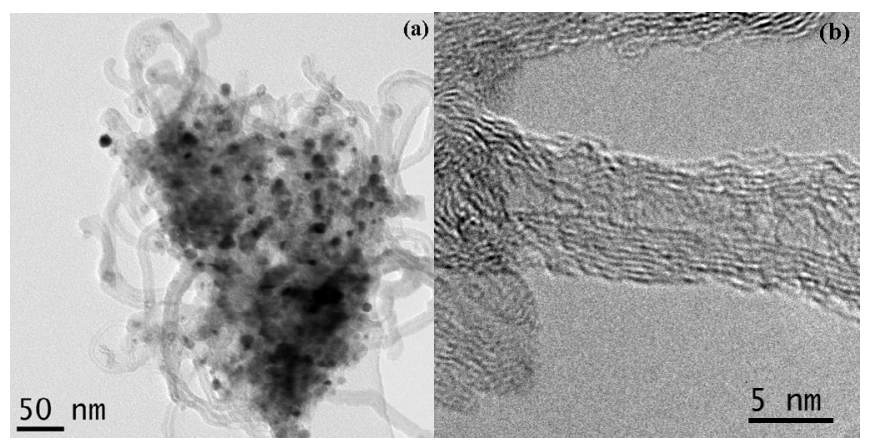

Figure 4: (a) Bright field image of a bundle of carbon nanotubes. (b) HRTEM of low temperature synthesised multi-walled carbon nanotube, showing graphitic planes aligned to nanotube axis.

Most of the nanotubes appear to be following a base-growth model [[14],[17],[18]], with the nanotube growing out of the catalyst particle and terminating with a closed end. The HRTEM image shows the graphene walls of the nanotubes, which are aligned to the nanotube axis. The graphene walls contain numerous defects, which are probably due to the low synthesis temperature.

This shows that nanotubes can be formed at low temperatures, using the combined effects of the plasma and a reduced hydrocarbon concentration. In plasma-assisted growth, the surface heating from the plasma is often ignored, or assumed to be similar to the set substrate temperature, as highlighted by Teo et al [[20]]. In our case, the temperature from plasma heating is monitored, without any intentional cooling of the substrate. This means that the heating is solely from the plasma. The results show that, despite the lower process temperature used, as compared to the usual temperatures reported, the synthesis of nanotubes is still possible. However, to achieve the growth of nanotubes without the lower defect levels typical for low temperature synthesis [[11]-[14]], a titanium layer is used here to allow an increase in the plasma heating of the surface, without also heating the substrate. In this work, the substrate temperature measured is a steady-state value. This can be seen from the SEM images, showing the effect of the applied bias, where the increasing applied bias reaches a state such that sufficient heating is attained for nanotube growth (Figure 2). With suitable thermal barrier material and a water cooled electrode as used in ref. [[11],[12]], the substrate temperature could be lowered further.

Using a 1D reactor model, Teo and co-workers ${ }^{[[20]]}$ proclaim in their paper that a simple parallel plate DC-PECVD reactor heats to approximately $700^{\circ} \mathrm{C}$ due to plasma heating, ignoring the reactor geometry and the resulting power flux on the electrode. This makes the comparison of different apparatus incorrect. Reactor geometry and coupled plasma power are the key parameters when considering substrate heating by the plasma; e.g. the DP800 system used in ref [11] has an exposed cathode area in excess of $500 \mathrm{~cm}^{2}$, translating to less than $0.5 \mathrm{~W} / \mathrm{cm}^{2}$ (i.e. $\sim 50^{\circ} \mathrm{C}[[20]]$ ). The power flux used by Teo and co-workers [[20]] was $20 \mathrm{~W} / \mathrm{cm}^{2}$ (i.e. $\sim 700^{\circ} \mathrm{C}$ ) whilst in this work, the power flux was $\sim 5 \mathrm{~W} / \mathrm{cm}^{2}$ (i.e. $\sim 350^{\circ} \mathrm{C}$ [[20]], similar to the highest temperatures we have measured during our growth). It is clear that low substrate temperatures and the growth of carbon nanotubes at relatively low substrate temperatures are possible.

The second factor contributing to the lowtemperature-growth is the reduced hydrocarbon concentration in the system. The lower synthesis temperature means that the arrival rate of the carbon at the surface has to be balanced with the diffusion process of the nucleation and growth. The importance of this balance was also reported by Minea and coworkers, where they adopted another approach by using an alternating nitrogen and acetylene plasma [[14]]. Obviously, if the surface or overall temperature is increased, more carbon can be introduced into the system due to the faster thermodynamically driven diffusion rate, as suggested by the use of $3.0 \%$ hydrocarbon at $550 \mathrm{~V}$ (Figure $3 \mathrm{~d}$ ).

The nanotubes synthesised using the plasma and the titanium layer predominately follow a base-growth mechanism, where the catalyst particle remains on the surface, as suggested by the TEM analysis. This is due to the strong interaction between the titanium buffer layer and the nickel catalyst. The entire growth process is thought to start with the breaking up and the fusing of the nickel particle with the titanium layer, thus forming a strong base support. At the initial stages of the growth, the carbon diffuses into/around the nickel catalyst. This continues until a super-saturation state is formed, following which, the carbon precipitates out of the nickel and the nanotube structure starts growing.

\section{CONCLUSION}

In conclusion, we show that it is possible to synthesise carbon nanotubes at low substrate temperatures, using a combination of plasma heating/dissociation and a titanium thermal buffer layer. The synthesised nanotubes have a lower level of defects than their typical low-temperaturegrown counterparts, which tends to produce carbon nanofibres. With a suitable substrate-cooling setup and a thermal barrier, it would be possible to achieve high growth rates for carbon nanotubes at room substrate temperatures, as well as low defect levels. 


\section{ACKNOWLEDGMENT}

The authors acknowledge the financial support from EPSRC in the form of a Carbon Based Electronics grant and the Portfolio Partnership Award.

\section{REFERENCES}

[1] Niu C., Sichel E.K., Hoch R., Moy D., Tennet H., Appl. Phys. Lett. 70, 1480 (1997)

[2] Tzeng Y., Chen Y., Liu C., Diamond Relat. Mater., 12, 774 (2003)

[3] Frackowiak E., Meternier K., Bertagna V., Beguin F., Appl. Phys. Lett. $77,1480(2000)$

[4] Liu T., Sreekumar T.V., Kumar S., Hauge R.H., Smalley R.E., Carbon 41, 2440 (2003)

[5] Teo K.B.K., Chhowalla M., Armaratunga G.A.J., Milne W.I., Hasko D.G., Pirio G., et al., Appl. Phys. Lett. 79, 1534 (2001)

[6] Wang Y., Rybczynsji J., Wang D.Z., Kempa K., Ren Z.F., Li W.Z., Kimball B., Appl. Phys. Lett. 85, 4741 (2004)

[7] Li J., Papadopoulos C., Xu J.M., Moskovits M., Appl. Phys. Lett. 75, 367 (1999)

[8] Franklin N.R., Wang Q., Tombler T.W., Javey A., Shim M., Dai H., Appl. Phys. Lett. 81, 913 (2002)

[9] Li J., Ye Q., Cassell A., Ng H.T., Stevens R., Han J., Meyyappan M., Appl. Phy. Lett. 82, 2491 (2003)

[10] Melechko A.V., Merkulov V.I., McKnight T.E., Guillorn M.A., Klein K.L., Lowndes D.H., Simpson M.L., Appl. Phys. Lett. 97, 041301 (2005)

[11] Boskovic B.O., Stolojan V., Khan R.U.A., Haq S., Silva S.R.P., Nat. Mater. 1, 165 (2002)

[12] Boskovic B.O., Stolojan V., Zeze D.A., Forrest R.D., Silva S.R.P., Haq S.J.. J. Appl. Phys. 96, 3443 (2004)

[13] Hofmann S., Kleinsorge B., Ducati C., Robertson J., New J. Phys. 5, $153.1(2003)$

[14] Minea T.M., Point S., Granier A., Touzeau M., Appl. Phys. Lett. 85, 1244 (2004)

[15] Englander O., Christensen D., Lin L., Appl. Phys. Lett. 82, 4797 (2003)

[16] Chhowalla M., Teo K.B.K., Ducati C., Rupesinghe N.L., Amaratunga G.A.J., Ferrari A.C., et al., J. Appl. Phys. 90, 5308 (2001)

[17] Tibbetts G.G., Devour M.G., Rodda E.J., Carbon 25, 367 (1987)

[18] Tibbetts G.G., J. Cryst. Growth 66, 632 (1984)

[19] Baker R.T.K., Carbon 27, 315 (1989)

[20] Teo K.B.K., Hash D.B., Lacerda R.G., Rupesinghe N.L., Bell M.S., Dalal S.H., Bose D., et al., Nano Lett. Vol 4, 921 (2004) 